\title{
CUTANEOUS MELANOMA, HODGKIN'S LYMPHOMA AND NON-HODGKIN'S LYMPHOMA: COMMON RISK FACTORS?
}

\author{
Mohamed Farouk Allam ${ }^{1,2}$, Pablo Fernández-Crehuet Serrano ${ }^{3}$, José Luis Fernández-Crehuet Serrano³, Khaled \\ Mahmoud Abd Elaziz ${ }^{2}$, Amparo Serrano Del Castillo ${ }^{1}$, Rafael Fernández-Crehuet Navajas ${ }^{1}$ \\ ${ }^{1}$ Department of Preventive Medicine and Public Health, Faculty of Medicine, University of Cordoba, Cordoba, Spain \\ ${ }^{2}$ Department of Community, Environmental and Occupational Medicine, Faculty of Medicine, Ain Shams University, Cairo, Egypt \\ ${ }^{3}$ Dermatology Department, Alto Guadalquivir Hospital, Andújar (Jaén), Spain
}

\section{SUMMARY}

Aim: An epidemiological cross-sectional study was conducted to evaluate the association between cutaneous melanoma, Hodgkin's lymphoma and non-Hodgkin's lymphoma in 40 European countries.

Methods: Incidence rates were obtained from the database of the International Agency for Research of Cancer (IARC). We analyzed age-adjusted and gender-stratified incidence rates for cutaneous melanoma, Hodgkin's lymphoma and non-Hodgkin's lymphoma in 40 European countries. All European countries included had registration systems that fulfilled the quality criteria of IARC. Normal distribution of the variables was examined using Kolmorov-Smirnov test before calculating their correlations using Pearson's Correlation test.

Results: In males, positive correlations were found between cutaneous melanoma, Hodgkin's lymphoma ( $r=0.14, p=0.38$ ), and non-Hodgkin's lymphoma $(r=0.64, p<0.001)$. In females, negative correlation was found between cutaneous melanoma and Hodgkin's lymphoma $(r=0.28$, $p=0.08)$, however, positive correlation was found between cutaneous melanoma and non-Hodgkin's lymphoma $(r=0.72, p<0.001)$.

Conclusion: Our findings raise the hypothesis about common risk factors for cutaneous melanoma and non-Hodgkin's lymphoma. New epidemiological and genetic studies are needed to identify possible common risk factors.

Key words: epidemiology, ecological, melanoma, non-Hodgkin's lymphoma, Hodgkin's lymphoma

Address for correspondence: M. F. Allam, Department of Preventive Medicine and Public Health, Faculty of Medicine, University of Cordoba, Avda. Menéndez Pidal, s/n Cordoba 14004, Spain. E-mail: fm2faahm@uco.es

\section{INTRODUCTION}

The most evident risk factors for cutaneous melanoma are environmental and life styles factors: exposure to ultraviolet (UV) radiation and geographic localization, together with individual factors such as number of nevi, dysplastic nevus, family history of melanoma, immune suppression, inability to get a tan, hypersensitivity to sun light, blue eyes, blond and red hair. In the general population the concomitant presence of 2 risk factors increases the risk of cutaneous melanoma by $2-4$ times, and the concomitant presence of 3 or more risk factors increases the risk by 20 times (1).

The International Agency for Research on Cancer (IARC) was established in 1965 by the World Health Assembly, as an independently financed organization within the structure of the World Health Organization (WHO). IARC develops research programmes to study cancer epidemiology and potential human carcinogens. One of its units prompts and coordinates cancer registration worldwide. The Cancer Incidence in Five Continents is a publication edited periodically by IARC since 1966 in collaboration with the International Association of Cancer Registries (IACR). This report includes information about cancer incidence in 5-year period. Information obtained from cancer registries worldwide should satisfy basic quality requirements. Before inclusion into the registries data are evaluated by the editorial committee of international experts (2).

Although ecological studies have several limitations and biases, they are considered simple studies and frequently used to investigate new health problems and exposures (3). These studies are exploratory and hypothesis generating, meanwhile experimental and analytical studies are considered confirmatory for these hypotheses. Advantages of these studies include availability of data and possibility to evaluate multiple levels of exposure in different geographic areas. Several authors like Susser suggest that ecological studies have several advantages because they investigate health problems in environmental context, as the health of the population is more than pooling of individual patients (4).

Previous study suggested that UV-induced impairment of immune functioning contributes to the aetiology of non-Hodgkin's lymphoma (NHL) (5). We suggest that cutaneous melanoma and non-Hodgkin's lymphoma could have the same risk factors (embryological origin of melanocytes and $\mathrm{T}$ lymphocytes, genetic factor or sun exposure).

The objective of the present study is to evaluate the association between cutaneous melanoma Hodgkin's lymphoma (HL) and NHL in 40 European countries. 


\section{MATERIALS AND METHODS}

An ecological study was conducted using the database of GLOBOCAN Project 2008 (IARC). Our study included the registries of 40 European countries: Albania, Austria, Belgium, Bosnia and Herzegovina, Bulgaria, Byelorussia, Czech Republic, Croatia, Cyprus, Denmark, Estonia, Finland, France, Germany, Greece, Hungary, Iceland, Ireland, Italy, Latvia, Lithuania, Luxemburg, Macedonia, Malta, Moldavian Republic, Montenegro, Netherlands, Norway, Poland, Portugal, Romania, Russia, Serbia, Slovakia, Slovenia, Spain, Sweden, Switzerland, Ukraine, and United Kingdom. All European countries included in our study had registration systems that fulfilled the quality criteria of IARC.

Of the mentioned registries, we utilized age-adjusted and gender-stratified incidence rates for the following types of cancer identified by their ICD 10: cutaneous melanoma (C43), Hodgkin's lymphoma (C81) and non-Hodgkin's lymphoma (C82-85, C96).

Normal distribution of the variables was examined using Kolmorov-Smirnov test before calculating their correlations using Pearson's Correlation test. Tests were considered significant for $p$ values $<0.05$. All statistical analyses were performed using the Statistical Package for Social Science (SPSS) version 15.0.

\section{RESULTS}

The mean incidence of cutaneous melanoma in males of the included European countries was $7.9 \pm 4.6$ per 100,000 inhabitants, with maximum incidence rates in Switzerland (18.1 per 100,000), Norway $(17.9$ per 100,000) and Sweden (16.1 per 100,000). Minimum incidence rates were reported in Cyprus (2.6 per 100,000), Greece (2.5 per 100,000) and Albania (2.2 per 100,000).

The mean incidence of cutaneous melanoma in females in the included European countries was $8.4 \pm 5.4$ per 100,000 inhabitants, with maximum incidence rates in Denmark (21.9 per 100,000), Switzerland $(20.5$ per 100,000$)$ and the Netherlands $(18.8$ per $100,000)$. Minimum incidence rates were reported in Montenegro (2.6 per 100,000), Greece (2 per 100,000) and Albania (1.7 per $100,000)$.

In males, positive correlations were found between cutaneous melanoma, $\mathrm{HL}(\mathrm{r}=0.14$ and $\mathrm{p}$ value 0.38$)$, and $\mathrm{NHL}(\mathrm{r}=0.64$ and $\mathrm{p}$ value $<0.001)$.

In females, negative correlation was found between cutaneous melanoma and HL ( $\mathrm{r}=-0.28$ and $\mathrm{p}$ value 0.08$)$, meanwhile positive correlation was found between cutaneous melanoma and NHL $(r=0.72$ and $p$ value $<0.001)$.

\section{DISCUSSION}

Currently, identification of NHL risk factors is the most crucial problem in the epidemiology of the disease. The present study aimed to evaluate the possible association between NHL and cutaneous melanoma.

Before reaching conclusions based on the present results, it is necessary to consider a number of potential objections to the methodology. Results of analyses base on ecological studies are considered methodologically imperfect by many investigators. Our results pertain solely to 40 European countries and therefore might not be generalizable. Other possible bias is the instability of calculated rates for a relatively rare disease like cancer. We should also take into account the cycle and oscillation of rates over time. We tried to avoid these possible biases including only cycle registration systems that fulfilled the quality criteria of IARC and WHO.

Our study has identified positive correlations between incidence of cutaneous melanoma and NHL ( $p$ value $<0.001$ ), which is in concordance with a study carried out on cancer registries in Australia, documenting the positive correlation between the increasing incidence of malignant melanoma and NHL in both men and women. This study suggested that exposure to high levels of ambient UV was shown to have positive correlation with the development of NHL (5).

In a study carried out in Australia on a large cohort of 18 thousand patients with malignant melanoma, about $0.3 \%$ of the cohort developed lymphomas. Almost $50 \%$ of diagnosed lymphomas occurred concurrently with malignant melanoma diagnosis. A striking finding was that $96 \%$ of the diagnosed lymphomas were NHL (6).

In another study on 664 patients with malignant melanoma in USA the incidence of NHL was 548 per 100,000 inhabitants, which was 16 times higher than in non-melanoma population (7).

The effect of migration from northern to southern part of the hemisphere also shows increased risk of development of both malignant melanoma and NHL, which denotes that higher exposure to UV could lead to the shift of increased risks for both cutaneous melanoma and NHL (5).

The previous finding of sunlight exposure as a risk factor for both malignancies was also documented in a study on Danish and Swedish cancer registries. This study showed that the relative risk of developing malignant melanoma after NHL was 2.4 (95\% confidence interval 1.8-3.2). The authors concluded that there was a strong probability that secular rise in exposure to ultraviolet light has increased the incidence rate of NHL (8).

Bidirectional association between NHL and cutaneous melanoma was found in a US study, which reported standardized incidence ratios of developing invasive cutaneous melanoma after NHL in men (1.31) and women (1.7). The authors concluded that sun exposure and/or depressed immunity could be common risk factors (9).

The correlation found between NHL and cutaneous melanoma raises the hypothesis about common risk factors for both neoplasms. This calls for further epidemiological and genetic studies to identify possible common risk factors for these two malignancies. Future studies should preferably be performed on large prospective cohorts to increase their internal validity.

\section{Conflicts of Interest}

None declared

\section{REFERENCES}

1. Cho E, Rosner BA, Colditz GA. Risk factors for melanoma by body site. Cancer Epidemiol Biomarkers Prev. 2005 May;14(5):1241-4.

2. World Health Organization, International Agency for Research on Cancer. IARC Scientific Publications Series [Internet]. Lyon: IARC [cited 2010 Oct 21]. Available from: http://www.iarc.fr/en/publications/list/sp/spub. php. 
3. Kleinbaum DG, Kupper LL, Morgenstern H. Epidemiologic research: principles and quantitative methods. Nueva York: Van Nostrand Reinhold; 1982.

4. Susser M. The logic in ecological: I. The logic of analysis. Am J Public Health. 1994 May;84(5):825-9.

5. McMichael AJ, Giles GG. Have increases in solar ultraviolet exposure contributed to the rise in incidence of non-Hodgkin's lymphoma? Br J Cancer. 1996 Apr;73(7):945-50.

6. Verwer N, Murali R, Winstanley J, Cooper WA, Stretch JR, Thompson $\mathrm{JF}$, et al. Lymphoma occurring in patients with cutaneous melanoma. J Clin Pathol. 2010 Sep;63(9):777-81.

7. Riou JP, Ariyan S, Brandow KR, Fielding LP. The association between melanoma, lymphoma, and other primary neoplasms. Arch Surg. 1995 Oct;130(10):1056-61
8. van der Aa MA, de Vries E, Hoekstra HJ, Coebergh JW, Siesling S. Sociodemographic factors and incidence of melanoma in the Netherlands, 1994-2005. Eur J Cancer. 2011 May;47(7):1056-60.

9. Balamurugan A, Rees JR, Kosary C, Rim SH, Li J, Stewart SL. Subsequent primary cancers among men and women with in situ and invasive melanoma of the skin. J Am Acad Dermatol. 2011 Nov;65(5 Suppl 1):S69-77.

Received September 3, 2014 Accepted in revised form April 17, 2015 